\title{
Le texte montre la langue, articule et disloque les discours
}

How the text discloses language, and both articulates and takes apart discourses

\section{Beth Brait}

Traducteur : Márcia Valéria Martinez de Aguiar

\section{OpenEdition Journals}

Édition électronique

URL : http://journals.openedition.org/aad/1186

DOI : 10.4000/aad. 1186

ISSN : 1565-8961

Éditeur

Université de Tel-Aviv

\section{Référence électronique}

Beth Brait, "Le texte montre la langue, articule et disloque les discours ", Argumentation et Analyse du Discours [En ligne], 7 | 2011, mis en ligne le 15 octobre 2011, consulté le 23 septembre 2019. URL http://journals.openedition.org/aad/1186 ; DOI : 10.4000/aad.1186

Ce document a été généré automatiquement le 23 septembre 2019.

\section{(1) $\$$}

Argumentation \& analyse du discours est mis à disposition selon les termes de la licence Creative Commons Attribution - Pas d'Utilisation Commerciale - Pas de Modification 4.0 International. 


\title{
Le texte montre la langue, articule et disloque les discours
}

How the text discloses language, and both articulates and takes apart discourses

\author{
Beth Brait
}

Traduction : Márcia Valéria Martinez de Aguiar

\section{NOTE DE L'ÉDITEUR}

Paru originellement en brésilien en 2008 [avec la date de 2007] dans la Revista de Filologia e Linguística Portuguesa 9 (São Paulo : Humanitas) sous le titre « O texto mostra a língua, costura e descostura discursos »

\section{Considérations initiales}

1 Je commencerai par une citation de Michel Foucault afin de saisir à travers le regard de ce philosophe un moment primordial de la manière dont le discours et son étude sont parvenus à occuper une position privilégiée, notamment depuis le 20e siècle, en se distinguant des études classiques sur la langue sans pour autant exclure celles-ci de leur corps de réflexion.

Dans son ouvrage Les mots et les choses, l'un des plus significatifs parmi une série d'écrits novateurs sur le langage dont il se fait qu'ils ont été tous été publiés en $1966^{1}$, Michel Foucault identifie le discours comme l'objet d'étude des Sciences humaines, distinguant cette perspective des autres formes de connaissance de la parole humaine :

bien que l'homme soit au monde le seul être qui parle, ce n'est point science humaine que de connaître les mutations phonétiques, la parenté des langues, la loi des glissements sémantiques; en revanche, on pourra parler de science humaine dès qu'on cherchera à définir la manière dont les individus ou les groupes se représentent les mots, utilisent leur forme et leur sens, composent des discours réels, montrent et cachent en eux ce qu'ils pensent, disent, à leur insu peut-être, 
plus ou moins qu'ils ne veulent, laissent en tout cas, de ces pensées, une masse de traces verbales qu'il faut déchiffrer et restituer autant que possible à leur vivacité représentative. L'objet des sciences humaines, ce n'est donc pas le langage (parlé pourtant par les seuls hommes), c'est cet être qui, de l'intérieur du langage par lequel il est entouré, se représente, en parlant, le sens des mots ou des propositions qu'il énonce, et se donne finalement la représentation du langage lui-même (1966 : 364).

3 Les effets de cette perspective foucaldienne sur le langage sont bien connus de différents courants de l'analyse du discours. Même si Foucault ne constitue pas pour tous un pôle d'ancrage obligé, l'extrait cité est assez révélateur, au même titre que bien d'autres formulations de l'auteur, d'une manière novatrice d'affronter la " masse de traces » verbales et extra-verbales qui habitent le texte et le projettent hors de lui, reliant socialement et historiquement sujet, vie et langage.

$\mathrm{Si}$, dans une perspective théorique, on peut considérer Les mots et les choses comme un ouvrage capital pour la constitution du discours en tant qu'objet des Sciences humaines, la littérature, à son tour, révèle depuis des siècles la manière dont les individus ou les groupes conçoivent les mots faisant du discours une arme puissante d'identité, d'insertion et d'intervention dans le monde. Gabriel García Márquez, dans son livre Vivre pour la raconter, écrit en exergue : «La vie n'est pas ce que l'on a vécu, mais ce dont on se souvient et comment on s'en souvient pour le raconter ». Le lien entre la vie, la mémoire et le discours s'explicite à chaque ligne de cette autobiographie, et parmi les diverses séquences qui concrétisent cette liaison, il y en a une qui sert particulièrement les intérêts de ce travail, puisqu'elle rend visibles les fils subtils qui articulent vie et langage :

On savait que l'agresseur s'exprimait avec l'accent doucereux des Andins, de sorte que le village exerça des représailles contre les nombreux étrangers si détestés qui parlaient avec un accent. Des bandes de villageois, armés de machettes à couper les régimes de bananes, se lançaient dans les rues la nuit tombée, attrapaient l'inconnu qu'ils surprenaient dans l'obscurité et lui ordonnaient de parler.

Selon l'intonation de sa voix ils le taillaient en pièces, sans même se rendre compte qu'il leur était impossible de ne pas se méprendre tant les accents étrangers étaient variés (Márquez 2003 : 56).

Cet extrait a en commun avec Les mots et les choses, nous semble-t-il, le fait d'expliciter la nécessité de reconnaître la dimension vitale assumée par le langage aussi bien en ce qui concerne les interventions de l'homme dans le monde que dans sa constitution en tant que sujet. La variation linguistique que retrace le texte de García Márquez fonctionne non seulement comme une empreinte régionale ou sociale, telle que l'analyserait la sociolinguistique, mais comme un ensemble de traces qui oppose des identités, dans une démonstration explicite d'intolérance. Cette intolérance, qui pourrait être religieuse ou politique, se concrétise en fait en tant qu'intolérance linguistique, matérialisée dans la violence physique rendue visible aussi bien dans les coups de machettes, qui accomplissent le carnage, que dans certaines expressions forgées par le narrateur, telles qu' « un accent doucereux » et « les nombreux étrangers si détestés ».

6 Ces deux expressions linguistiques sont indicatives du lieu d'où parlent les sujets en question, des discours qui les traversent et qui les font agir de façon aussi violente. La présence de ces expressions dans le texte permet une réflexion discursive qui, pour être menée, doit prendre appui sur l'ensemble du récit, et sur les lieux historiques et sociaux qu'elles convoquent. La langue en usage, réactivée en l'occurrence par la 
mémoire d'un écrivain, dénie tout en montrant les discours qui la constituent. C'est au lecteur, armé de ses conquêtes théoriques, de considérer cette "masse de traces verbales qu'il faut déchiffrer et restituer autant que possible à leur vivacité représentative ", selon la suggestion de Michel Foucault.

De la perspective des théories et des analyses du discours, il est donc possible de surprendre discursivement la manière dont sont constituées, dans les textes, la force brute, l'intolérance, l'inégalité, par exemple, comme les dépeint Vivre pour la raconter. Mais ce sont également les articulations singulières de la diversité linguistique qui révèlent les rencontres et la cohabitation de l'inégal et du pluriel, comme on le verra par la suite. C'est en ce sens que l'analyse du discours participe des Sciences humaines et qu'elle devient, à partir du 20e siècle, un domaine de construction des connaissances, constituée par des courants ${ }^{2}$ aussi divers qu'originaux. Cependant, indépendamment de tout courant, l'importance de l'analyse du discours réside dans le fait qu'elle permet de rechercher et de souligner les richesses et les misères que le langage porte en lui en tant qu'histoire, culture, constitution, emprisonnement et libération des sujets.

\section{Sphère et relations dialogiques : deux concepts centraux de la pensée bakhtinienne}

Dans cet article, nous voudrions souligner quelques aspects qui relèvent d'un de ces courants - l'analyse dialogique du discours ${ }^{3}$. Bien qu'il n'ait jamais été ainsi nommé formellement, ce courant peut être reconnu ou reconstitué à partir des contributions incontestables de la pensée bakhtinienne aux études du langage. Sans se présenter comme un ensemble de préceptes systématiquement organisés pour fonctionner comme une perspective théorique et analytique close, les ouvrages de Bakhtine, Voloshinov, Medvedev et autres participants de ce qu'à présent on désigne par Cercle de Bakhtine trouvent leurs assises dans une conception du langage, de la construction et de la production du sens, qui s'appuie nécessairement sur les relations discursives mises en place par des sujets historiquement situés ${ }^{4}$. Et, dès les premiers textes parus dans les années 1920, contrairement à ce que l'on croit communément, ces études indiquent certaines voies méthodologiques, comme on peut le vérifier dans les passages suivants, extraits de La poétique de Dostoïevski et de Marxisme et philosophie du langage.

Nous avons intitulé notre chapitre "Le mot chez Dostoïevski ", car nous nous y attacherons à l'étude de la langue dans sa totalité concrète, vivante, et non pas de la langue comme objet spécifique de la linguistique, obtenu en faisant abstraction de certains côtés de la vie concrète du mot (ce qui en linguistique était parfaitement légitime et même nécessaire). Et ce sont précisément ces côtés concrets qui pour nous présentent un intérêt capital. Par conséquent, nos analyses ultérieures ne seront pas linguistiques, dans le sens exact du terme. On peut les rattacher à la translinguistique, si on entend par celle-ci une science qui ne serait pas encore strictement déterminée par des disciplines précises, bien délimitées, et consacrée à ces aspects du mot qui sortent du cadre de la linguistique. Il est évident que dans ses recherches, la translinguistique ne peut ignorer la linguistique et doit se servir des résultats obtenus par cette dernière. Toutes les deux étudient le même phénomène concret, infiniment complexe et multiforme: le mot, mais elles en choisissent divers aspects et les observent sous des angles différents. Elles doivent se compléter, non se mélanger. Dans la pratique leurs frontières sont souvent difficiles à respecter (Bakhtine $1970: 238$ ). 
C'est précisément là, dans l'échange verbal concret, que le langage vit son devenir historique, et non dans le système linguistique abstrait des formes de langues, pas plus que dans le psychisme individuel des lecteurs [en italique dans le texte russe].

Il en découle que l'ordre méthodologique à suivre pour étudier le langage doit être le suivant: (1) les formes et les types d'interactions concrètes dans lesquelles ces interactions se réalisent; (2) les formes des énoncés singuliers, des interventions verbales singulières en liaison étroite avec l'interaction dont ils font partie, autrement dit les genres d'interventions verbales dans la vie et dans la création idéologique déterminés par l'interaction verbale ; (3) à partir de là, un réexamen des formes linguistiques telles qu'elles sont habituellement interpretées (Vološinov $2010: 321)$.

9 Et c'est dans le cadre de cette perspective discursive qu'a été choisi, pour aborder les textes et les discours qui y circulent, le concept de "sphère ", défini de la façon suivante dans Marxisme et philosophie du langage ${ }^{5}$ :

À l'intérieur même du domaine des signes, autrement dit dans le domaine idéologique, il existe de grandes différences: en effet, en font partie aussi bien l'image artistique que le symbole religieux, la formule scientifique, la norme juridique, etc. Chaque domaine de la création idéologique s'oriente à sa manière dans la réalité et la réfracte à sa manière. Chaque domaine a sa fonction spécifique dans l'unité de la vie sociale. Mais c'est le fait d'être un signe qui constitue la caractéristique commune à tous les phénomènes idéologiques (Vološinov 2010 : 131) [en italique dans le texte russe].

10 Ce concept, que l'on retrouve également dans d'autres ouvrages du Cercle $^{6}$, est obligatoirement lié à la perspective de la production, de la circulation et de la réception des textes et des discours et, particulièrement, au concept de "relations dialogiques ». Les relations dialogiques, notion centrale de la pensée bakhtinienne, s'établissent à partir des « sphères » discursives et des événements qui y sont impliqués. Ce sont elles qui produisent les regards, les lieux discursifs, les sens et donc la connaissance. Ces relations sont ainsi définies dans La poétique de Dostoïevski :

Les rapports dialogiques ne se laissent réduire ni aux rapports logiques ni aux rapports de signification objective que sont en eux-mêmes dépourvus de moment dialogique. Les seconds doivent se couler dans le mot, devenir des énoncés, exprimer par les mots de différents sujets, pour que des rapports dialogiques puissent naître entre eux (Bakhtine 1970 : 240).

11 Les rapports dialogiques ne sont pas seulement possibles entre énoncés complets (relativement), mais peuvent s'établir à l'égard de toute partie signifiante de l'énoncé, même à l'égard d'un mot isolé, si celui-ci est perçu en tant que mot impersonnel de la langue, mais en tant que signe de la position interprétative d'autrui, en tant que spécimen de son énoncé, c'est-à-dire si l'on y entend une voix autre. C'est pourquoi les rapports dialogiques peuvent pénétrer à l'intérieur même de mots isolés, à condition que deux voix s'y affrontent dialogiquement (Bakhtine $1970: 241-242$ ).

\section{Vir-se [se venir] : des langues differentes?}

Sans trop insister sur les questions théoriques, mais cherchant à les saisir dans les pratiques sociales et discursives choisies, il est important de souligner que les "relations dialogiques» ne sont jamais données a priori. Au contraire, elles sont établies à partir d'une énonciation, d'une interaction, d'une sphère ou de l'affrontement des sphères, par un énonciateur, par un sujet qui les signe; elles impliquent des interlocuteurs et leurs différences. Loin donc d'être naturelles, elles 
s'établissent à partir d'un point de vue idéologique, théorique ou bien artistique, comme dans le cas des vers qui voici :

Estou a vir/ Je suis en train de venir

e tu como é que tens por dentro ?/ et toi comment te sens-tu dedans?

por que não te vens também ?/ Pourquoi ne te viens-tu [sic] pas aussi ?

13 Cette strophe ainsi transcrite, dépourvue de toute signature et hors de tout contexte, comme si elle avait été extraite arbitrairement d'un poème, réclame un contexte, quelque chose qui puisse permettre à son destinataire de la saisir, de se l'approprier et d'émettre des hypothèses sur ses sens possibles. Peut-être cela ne s'applique-t-il pas aux locuteurs du portugais du Portugal, connaissant les variantes de la langue portugaise parlée au Portugal. Il est alors possible que le destinataire ait tout de suite identifié la signification, dans le sens bakhtinien du terme, de chacun des vers, de la syntaxe textuelle et discursive qui les articule et qu'il ait donc saisi la signification de la strophe. Cependant, même dans ce cas-là le destinataire se pose la question du sens, puisque la strophe, déliée de tout contexte et de signature, produit en tant que thème une interrogation, et non pas nécessairement un sens possible ou des effets de sens possibles.

14 Pour les autres, pour ceux qui ne dominent pas les variations du portugais du Portugal, un certain aspect linguistique se manifeste d'emblée, donnant lieu à une interrogation: si, d'un côté, l'emploi du tu [tu] et de l'expression estou a vir [je suis en train de venir] à la place du gérondif estou vindo, plus usuel dans le portugais du Brésil, peut nous renvoyer à des connaissances générales et stratifiées du portugais du Portugal, c'est cependant l'emploi inusité, au moins pour les Brésiliens, du verbe vir [venir] dans deux des trois vers, qui pointe vers quelque part et, en même temps, vers nulle part: « estou a vir » [je suis en train de venir] dans le premier vers; et "por que não te vens » [pourquoi est-ce que tu ne te viens pas] dans le troisième. Comment résoudre, bien que partiellement, cette interrogation? Quel est ce lieu vers où pointe le verbe vir [venir] ?

D'abord il faut savoir que cet ensemble de vers apparaît dans un contexte particulier. Il s'agit de l'une des pistes du disque C $\hat{e}^{7}$, de Caetano Veloso, plus précisément la piste 10, Por quê? [Pourquoi ?], composée uniquement de ces trois vers.

L'écoute de Por quê? [Pourquoi ?] offre une autre donnée, par rapport à la simple lecture des vers, suggérant une interprétation possible ou au moins quelques effets de sens qui auront des conséquences linguistiques importantes. L'accent du portugais du Portugal choisi par l'interprète, qui est aussi l'auteur de la chanson, indique un lieu de vie de la langue portugaise qui n'est pas le Brésil, mettant ainsi en place au moins une dualité de voix, un plurilinguisme: d'un côté, l'articulation paroles-musique, que permettent les particularités de la chanson en tant que texte circulant dans la sphère artistique musicale. De l'autre, un chanteur brésilien connu, parlant donc le portugais du Brésil, Caetano Veloso, qui simule la manière de parler du Portugal pour interpréter sa chanson. Il s'agit donc d'une chanson composée par un Brésilien qui l'interprète avec un accent d'outre-Brésil, indiquant un événement discursif constitutif d'une "autre langue ", d'une autre culture, mais qui circule dans une sphère brésilienne.

17 C'est à partir de ces données que s'ouvrent des directions de recherche sur les significations de la chanson et de quelques-uns de ses sens et effets de sens possibles. La question devient alors plus explicite: quelle pratique discursive est récupérée, réinstaurée par les paroles de cette chanson? Ceux qui dominent le portugais du Portugal ne se sont sans doute pas même posé cette question, parce qu'ils ont tout de 
suite compris la pratique discursive et sociale qui a produit la chanson. Cependant, les Brésiliens ont besoin de ce référent discursif et social, qui échappe complètement à leur vécu linguistique et culturel.

On a tout loisir de se rapporter au dictionnaire, ce puissant outil linguistique, pour y chercher les significations du verbe vir [venir] en tant que pronominal, car même s'il ne fait pas partie du lexique actif des brésiliens, l'accent choisi par l'interprète signale cependant qu'il appartient au lexique du portugais du Portugal. Et en effet, dans le Dicionário eletrônico Houaiss da Língua Portuguesa, on retrouve dans l'acception 26 de l'article vir [venir] : verbe vir, pronominal Portugal : atteindre l'orgasme.

Tout devient clair. Le poète/troubadour a apporté pour les Brésiliens, dans la sphère musicale, la possibilité de connaître, sous un autre angle, sous un nouveau regard, dans une nouvelle dimension linguistique et discursive, ce que l'on connaît si bien dans la pratique : atteindre l'orgasme, identifié d'une manière complètement nouvelle.

$20 \mathrm{Si}$, d'un côté, le dictionnaire explique la signification du verbe vir pronominal, montrant la richesse des variations et des possibilités d'une langue, il reste cependant encore à savoir à quels discours cette transposition d'une sphère à une autre donne lieu. Il n'est pas sans intérêt d'aller un peu plus loin pour savoir comment la recherche des significations va, également, révéler les discours qui traversent les sujets qui écoutent la chanson et, chose très importante, comment elle va dévoiler des formes de production de sens ou des effets de sens, explicitant, en différentes interactions, les relations langue-discours en circulation, en tant que points de vue à partir desquels les sujets se révèlent ou révèlent leur idéologie, leurs valeurs.

21 La lecture d'un entretien de Caetano Veloso, plus précisément du passage où il parle de la chanson Por quê ? peut contribuer à illustrer cette perspective. Lors de l'entretien, le compositeur-interprète essaie d'expliquer la signification de la chanson à partir du verbe vir-se. Comme on verra, il fera de curieuses observations linguistiques, commentant entre autres l'interprétation à son avis "erronée " d'un critique qui, écoutant l'accent du Portugal, a assimilé la chanson à « une blague ».

Mais non, la chanson est sérieuse, sur un sujet sérieux et, pour moi, elle a encore plus de valeur en raison de l'observation linguistique. C'est que nous, Brésiliens, employons le verbe « gozar», comme les français emploient le verbe «jouir », dans le sens d'atteindre l'orgasme. Ces verbes se rapportent à la volupté, à la jouissance du moment de l'orgasme, alors que leur équivalent en anglais, « to come » semble le point final de quelque chose, ainsi que l'espagnol «acabar». Mais les Portugais l'emportent sur ce point, car ils emploient le verbe "vir» [venir] dans sa forme réflexive : "vir-se » [se venir]. La personne se vient. Je trouve cela très beau. Et le critique en question n'a pas pu s'empêcher de penser qu'il s'agissait d'une blague, parce qu'il croit que tout ce qu'on dit avec un accent du Portugal ne peut être qu'une blague. C'est l'interprétation la plus vulgaire qu'une chanson aussi peu vulgaire ait pu trouver (Veloso $2006: 86$ ).

Ces textes - celui de la chanson qui circule dans la sphère artistique, celui du critique et celui de l'auteur, qui circulent dans la sphère des médias - nous aident à penser quelques aspects de la complexité et de la richesse produites par les intersections entre langue, sphères de circulation et discours circulant.

23 Ainsi, un auteur, un créateur de chansons brésiliennes, qui parle donc le portugais du Brésil, découvre une expression caractéristique d'une pratique discursive usuelle chez les Portugais - vir-se [se venir] - plus précisément la manière dont le portugais du Portugal traduit linguistiquement une particularité du rapport sexuel - puis se laisse 
séduire par cette expression linguistique, au point de la transporter dans une autre sphère, à savoir la sphère artistique où circule la chanson. Cette transposition fonctionne sans aucun doute comme une forme discursive qui réoriente le regard culturel des Brésiliens. Ce qui était discours du quotidien d'une fraction de la culture portugaise dans des conditions très marquées, devient discours artistique de la culture brésilienne à partir d'un déplacement qui ré-accentue l'expression et la transpose dans d'autres conditions socio-historiques.

Ce passage ne saurait se faire d'une manière tranquille, sans conséquences discursives, sans jugements de la part des sujets concernés. Il suffit d'observer le sentiment d'étrangeté qui saisit les Brésiliens à l'audition de cette chanson et qui les amène, par exemple, à consulter un dictionnaire de la langue portugaise. Ou encore, la réaction du critique qui offre immédiatement sa version, fondée sur le seul accent du Portugal adopté par l'interprète, ce qui, à ses yeux, ne peut être que l'indice d'une «blague ». Et, enfin, le besoin ressenti par l'auteur de construire une explication a posteriori. Le passage d'une expression ordinaire de la langue portugaise pratiquée au Portugal, véhiculé habituellement dans la sphère du quotidien, à la langue portugaise pratiquée au Brésil, accueillie dans la sphère artistique musicale, révèle non seulement la complexité linguistique et la charge de signification de l'expression, mais aussi l'amplification discursive provoquée par la nouvelle sphère et les relations dialogiques différenciées qui s'y instaurent.

On s'aperçoit, par exemple, que quand elle provoque de l'étrangeté chez ses auditeurs brésiliens et chez le critique lui-même - partant un auditeur averti - la chanson pointe, avant même l'explication de son auteur, vers des lieux discursifs et socio-historiques qui se situent hors d'elle-même, mais ne sont pas tout à fait extérieurs à la langue portugaise et aux discours dans lesquels ses locuteurs sont plongés. C'est comme si la chanson réclamait un sens, la ré-orientation du regard, de l'oreille, et que les interlocuteurs répondaient du lieu où ils se situent, au moyen des discours qui les traversent et qui traverseront désormais la chanson.

Ces lieux permettent de retrouver, à partir du point de vue de la langue, de la sphère où l'expression a été mise en circulation et des discours articulés et disloqués par l'usage, la manière dont deux cultures différentes présentent des perspectives qui créent des dimensions diverses pour une même "pratique sociale " - il y faut les guillemets - et pour une "même langue». Le regard de la langue, le regard des langues en convergence, le regard de l'auteur, le regard du critique, les différents regards des langues, récupérées dans l'explication de l'auteur, permettent de mesurer la différence, la variété, le dissemblable crée par la variété.

Tout en expliquant, à la manière du linguiste, dans la sphère des médias, les différentes formes qu'un aspect de la pratique sexuelle reçoit en différentes langues, révélant des points de vue culturels différents sur ce que l'on croit être absolument identique dans toutes les cultures, Caetano Veloso présente aussi ses jugements, en tant que commun des mortels, usager et appréciateur des parlers du monde, en laissant entrevoir les discours qui le constituent en tant que sujet historique, social, culturel. En ce sens, il s'éloigne du regard du linguiste pour faire des évaluations esthétiques, comme il est naturel chez un artiste. Mais il ne pourrait exprimer aucune de ses opinions dans la sphère artistique et musicale, sous peine de transformer la chanson en un autre texte : un manifeste, peut-être. 

traces explicites ou dissimulées qu'elle laisse dans les textes, il est possible de comprendre les relations inaliénables du langage et de la vie. Si le texte montre la langue, articule et disloque les discours, c'est à l'analyse du discours de le fouiller en quête des «traces" de natures diverses qu'il faut "déchiffrer et reconstituer ». Il ne s'agit pas cependant d'atteindre la vérité, le véritable sens d'un texte ou d'un groupe de textes. Loin de là, comme nous avons essayé de le montrer, les sens et les effets de sens produits par un texte ne peuvent être identifiés qu'à partir de certains concepts issus des fondements théoriques qui permettent de le lire. La distinction conceptuelle entre "pratiques discursives» et "pratiques sociales » dépend sans aucun doute du lieu théorique qui les postule. De même, les concepts de sphère et de relations dialogiques ont un statut particulier dans la pensée bakhtinienne, dans le cadre de ce qu'on peut appeler analyse/théorie dialogique du discours, permettant un travail de compréhension des formes de production de sens et des effets de sens.

C'est à travers le prisme de ces concepts qu'un même énoncé, véhiculé en différentes sphères, en différentes cultures, devient autre. Qu'un même texte devient autre.

\section{BIBLIOGRAPHIE}

Bakhtine, Mikhaïl. 1970 [1e édition russe 1963]. La poétique de Dostoïevski. Traduit de russe par Isabelle Kolitchef (Paris : Seuil)

Argumentation et Analyse du Discours, 7 | 2011 
Bakhtine, Mikhaill. 1978 [1e édition russe 1975]. Esthétique et théorie du roman. Trad. Daria Olivier (Paris : Gallimard)

Bakhtine, Mikhaïl. 1984 [1e édition russe 1979]. Esthétique de la création verbale. Trad. Alfreda Aucouturier. Preface Tzvetan Todorov (Paris : Gallimard)

Bajtín, M. \& V. Voloshinov. 1997 [1e édition russe 1926]. « La palabra en la vida y la palabra en la poesia », Hacia una filosofía del acto ético. De los borradores. Y otros escritos. Trad. Tatiana Bubnova (Barcelona : Anthropos Editorial), 106-137

Brait, Beth. 2006. « Análise do discurso », Brait, B. (éd.). Bakhtin outros conceitoschave (São Paulo : Contexto), 9-32

Brait, Beth. 2006a. « Uma perspectiva dialógica de teoria, método e análise », Gragoatá 20, 47-62

Foucault, Michel. 1966. Les mots et les choses (Paris : Gallimard)

Grillo, Sheila Vieira de Camargo. 2006. «Esfera e campo », Brait, B. (éd.) Bakhtin outros conceitoschave (São Paulo : Contexto), 133-160

Marquez, Gabriel García. 2003. Vivre pour la raconter. Trad. Annie Morvan (Paris : Grasset)

Medvedev, P. N. Cercle de Bakhtine. 2008 [1e édition russe 1928]. La méthode formelle en littérature. Introduction à une poétique sociologique. Trad. Bénedicte Vauthier et Roger Comtet (Toulose : P. U. du Mirail)

Veloso, Caetano. 2006. RG VOGUE 55

Veloso, Caetano. 2006. Por quê ?Cêe (Production Universal Music sous la direction de Pedro Sá et de Moreno Veloso)

Vološinov, Valentin Nicolaevič 2010 [1e édition russe 1929]. Marxisme et philosophie du langage. Les problèmes fondamentaux de la méthode sociologique dans la science du langage. Trad. Patrick Sériot \& Inna Tylkowski-Ageeva (Limoges : Lambert-Lucas)

\section{NOTES}

1. Je pense ici aux Problèmes de linguistique générale I d'E. Benveniste, à La sémantique structurale, d'A. J. Greimas et aux Ecrits de J. Lacan, études publiées en 1966.

2. Je me réfère ici à la pluralité des tendances issues des diverses sources théoriquesméthodologiques, qui se distinguent par leur «accent». On reconnaît en général les accents français, russe, anglo-saxon ou encore, en certains cas, le mélange de deux accents ou plus, ce qui met nécessairement en place une nouvelle variante.

3. J'ai examiné plus en détails les caractéristiques de cette tendance dans « Análise et teoria do discurso » [Analyse et théorie du discours], (Brait [éd.] 2006 : 9-32).

4. Quelques aspects théoriques et surtout méthodologiques de cette analyse/théorie du discours ont été précisés dans mon article "Uma perspectiva dialógica de teoria, método e análise" [Une perspective dialogique de théorie, de méthode et d'analyse], (Brait 2006a : 47-62).

5. Voir à propos du concept de sphère, l'article « Sphère et champ » (Grillo, 2006).

6. On peut également retrouver le concept de sphère dans Bajtín \& Voloshinov 1997, Medvedev 2008, Bakhtine 1978.

7. Cê. Production Universal Music sous la direction de Pedro Sá et Moreno Veloso, 2006 


\section{RÉSUMÉS}

Cet article cherche à analyser la manière dont une certaine forme linguistique, transplantée d'une sphère de circulation à une autre, d'une pratique discursive à une autre, d'une culture à une autre, fait naître des discours différenciés, qui révèlent la richesse de la langue et sa productivité discursive dans des contextes différents. Fondé sur les concepts de sphère et de relations dialogiques issus de la pensée de Bakhtine, et considérant que les études du langage mises en œuvre par les différents courants de l'analyse du discours participent d'une même tentative de comprendre, dans sa dimension historique, sociale et culturelle, la production de sens, ce travail a comme principal objet l'examen des vers d'une chanson brésilienne contemporaine, dans laquelle le verbe vir [venir] est employé dans sa forme pronominale vir-se [se venir]. L'observation de la présence de cette forme totalement étrangère aux locuteurs du portugais du Brésil, dans une sphère artistique brésilienne, permet de reconnaître, entre autres, les formes de la langue en usage en tant que partie de l'objet visé par les analyses et les théories du discours.

This paper analyzes a language structure which, transferred to a different sphere, a different discursive practice, and a different culture, leads to differing discourses which, in turn, point to the Portuguese language assets and to its discursive productivity in different contexts. Our work is supported by the concepts of sphere and dialogical relations, both discussed in Bakhtinian terms, and takes into account the fact that language studies practiced by several trends of Discourse Analysis may boil down to the attempt to understand, by means of historical, social and cultural dimensions, the production of meaning. This paper mainly aims to study the verses of a Brazilian contemporary song in which the verb vir ("to come") is used in its pronominal form vir-se ("to come" + pron.). Scrutinizing the use of this form - which is totally foreign to Brazilian speakers of Portuguese - within the Brazilian artistic realm, one is lead to recognize the forms of language in use as part of the object targeted by analyses and by theories of discourse.

\section{INDEX}

Keywords : contemporary song, discourse, language, sphere

Mots-clés : chanson contemporaine, discours, langue, sphère

\section{AUTEURS}

BETH BRAIT

Pontifícia Universidade Católica de São Paulo 